\title{
Cell Therapy for Spinal Cord Injury by Neural Stem/Progenitor Cells Derived from iPS/ES Cells
}

\author{
Osahiko Tsuji • Kyoko Miura • Kanehiro Fujiyoshi • \\ Suketaka Momoshima • Masaya Nakamura • \\ Hideyuki Okano
}

Published online: 10 September 2011

(C) The American Society for Experimental NeuroTherapeutics, Inc. 2011

\begin{abstract}
Reports of functional recovery from spinal cord injury after the transplantation of rat fetus-derived neural stem cells and embryonic stem cells has raised great expectations for the successful clinical use of stem cell transplantation therapy. However, the ethical issues involved in destroying human embryos or fertilized oocytes to obtain stem cells have been a major obstacle to developing clinically useful stem cell sources, and the transplantation of stem cells isolated from other human embryonic tissues has not yet been developed for use in clinical applications. Recently, induced pluripotent stem cells, which can serve as a source of cells for autologous transplantation, have been attracting a great deal of attention as a clinically viable alternative to stem cells obtained directly from tissues. In this review, we outline the
\end{abstract}

Electronic supplementary material The online version of this article (doi:10.1007/s13311-011-0063-z) contains supplementary material, which is available to authorized users.

\author{
O. Tsuji $\cdot$ K. Fujiyoshi $\cdot$ M. Nakamura $(\bowtie)$ \\ Department of Orthopaedic Surgery, \\ School of Medicine, Keio University, \\ 35 Shinanomachi, Shinjuku, \\ Tokyo 160-8582, Japan \\ e-mail: masa@sc.itc.keio.ac.jp \\ O. Tsuji $\cdot$ K. Miura $\cdot$ K. Fujiyoshi $\cdot$ H. Okano $(\bowtie)$ \\ Department of Physiology, School of Medicine, Keio University, \\ 35 Shinanomachi, Shinjuku, \\ Tokyo 160-8582, Japan \\ e-mail: hidokano@a2.keio.jp \\ S. Momoshima \\ Department of Radiology, School of Medicine, Keio University, \\ Tokyo, Japan \\ K. Fujiyoshi \\ Department of Orthopaedic Surgery, Murayama Medical Center, \\ National Hospital Organization, \\ Tokyo, Japan

neural induction of mouse embryonic stem cells and induced pluripotent stem cells, their therapeutic efficacy in spinal cord injury, and their safety in vivo.

Keywords Induced pluripotent stem cells · Embryonic stem cells $\cdot$ Neural differentiation $\cdot$ Cell transplantation $\cdot$ Spinal cord injury · Safety

\section{Introduction}

At the start of the 20th century, Spanish neuroanatomist Santiago Ramón y Cajal [1] wrote, "Once the development was ended, the fonts of growth and regeneration ... dried up irrevocably," neatly articulating the long unchallenged theory that neurogenesis does not occur in adult mammals and that, once injured, the adult mammalian CNS, especially the spinal cord, can never regenerate. However, in 1992, cells with the potential to generate new neurons, or neural stem cells, were identified by Reynolds and Weiss [2]. Subsequently, methods were established to culture mammalian neural progenitor cells, including stem cells, from animals and humans [3-5].

When the molecular biological properties of neural stem cells in the developing and adult CNS were elucidated [610], attempts were made to take advantage of their pluripotency to regenerate nervous system cells and tissues that had been lost as a result of neurodegenerative disease or injury. While being maintained in an undifferentiated state, neural stem cells divide and proliferate. These cells possess the potential for self-renewal (i.e., for giving rise to cells that are identical to themselves) and for pluripotency (i.e., giving rise to neurons, astrocytes, and oligodendrocytes), the 3 cell types that comprise the nervous system. Moreover, they can be cultured in vitro by a variety of methods, including being cultured as spherical bodies by 
the neurosphere method, although these spheres mainly contain the progenitor cells, and the stem cell content within the sphere is actually quite low. Therefore, as many neural stem/progenitor cells as needed can be generated in culture, and these cells show promise for transplantation cell therapy, particularly in spinal cord injury (SCI) research, because the transplantation of fetal spinal cord tissue leads to functional recovery from SCI in the rat [11].

After this report, studies were conducted in the authors' own laboratories to establish the safety and efficacy of transplanting in vitro expanded stem/progenitor cells to treat SCI. We tested the efficacy of transplanting: 1) rat fetal spinal cord-derived neural stem/progenitor cells to treat rat SCI during the subacute stage after injury [12], 2) mouse fetal striatum-derived neural stem/progenitor cells to treat SCI in mice (an experiment that used luciferase luminescence to bioimage the transplanted cells) [13], and 3) human fetal brain-derived neural stem/progenitor cells to treat SCI in a nonhuman primate, the common marmoset (Callithrix jacchus), as part of a pre-clinical trial [14]. We found good functional recovery in all 3 cases and reported it ref [12-14]. These results led to strong expectations that human neural stem/progenitor cells that had been cultured and expanded in vitro would be applied to nerve regeneration in humans. However, the fact that these cells always had to be collected from the brains of aborted fetuses has been a major factor in precluding the clinical use of human neural stem/progenitor cells, and even now there are still no prospects for their clinical application in Japan.

In recent years, induced pluripotent stem (iPS) cells that possess embryonic stem (ES) cell-like pluripotency and proliferative capacity have been produced by transducing several different genes into somatic cells [15-17]. If it were possible to perform custom cell transplantation therapy by generating iPS cells from patients themselves and transplanting them into SCI sites after the iPS cells had been induced to differentiate into neural stem/progenitor cells, it would also be possible to avoid both the ethical problem of using human fetal tissue and the possibility of immunological rejection. In this article, we will outline the in vitro culture methods of inducing ES cell- and iPS cell-derived neural stem/progenitor cells, discuss their safety at transplantation, and describe current research on their transplantation into SCI models in vivo.

\section{ES Cell-Derived Neural Stem Cells}

\section{In Vitro Models of Neural Development and Mouse ES Cell-Derived Neural Stem Cells}

As previously stated, neural stem cells are defined as having self-renewal potential and pluripotency. However, their ability to differentiate and proliferate is strictly governed by their time and place of birth, and not all neural stem cells have the exact same properties. Neural stem cells are already present on approximately embryonic day 5 and can be cultured in the presence of leukemia inhibitory factor [18]. Soon thereafter, and still at a relatively early stage of embryonic development (embryonic days 8.5-12.5), the neural stem cells can be cultured in vitro in the presence of fibroblast growth factor-2 (FGF-2). From this stage until the late stage of embryonic development, the radial glia located around the cerebral ventricle possess the properties of neural stem cells, self-renewing by symmetric cell division and producing neurons by asymmetric cell division $[19,20]$. In the late stage of embryonic development, neural stem cells proliferate in vitro in response to epidermal growth factor, as well as FGF-2. However, the neural stem cells that emerge from this stage onward are no longer able to produce early-born projection neurons, such as forebrain-type cholinergic neurons, dopaminergic neurons, or motor neurons, which are born only in the early stage of development. Finally, from the late stage of embryonic development through the neonatal period and into adulthood, neural stem cells in the brain are mainly present around the cerebral ventricles and produce glial cells (astrocytes and oligodendrocytes), as well as neurons [21]. Thus, the differentiation and proliferation capacity of neural stem cells is strictly controlled by the spatial and temporal specificities that the cells are endowed with at the time and place where they are born.

In a report from our group, Okada et al. [22] used mouse ES cells derived from the inner cell mass to induce neural stem/progenitor cells with high plasticity that are present in a relatively early stage of development (i.e., they succeeded in constructing a neural development model culture system in vitro that mimics neural development and reflects its temporal and spatial specificity). This culture system eliminates the requirement for leukemia inhibitory factor to maintain the ES cells in their undifferentiated state, and by using floating cultures it allows the formation of embryoid bodies (EBs), which contain cells derived from the 3 germ layers. EBs contain relatively early-stage neural stem/ progenitor cells, and by growing EBs in floating cultures containing serum-free neural stem cell culture medium supplemented with FGF-2, it is possible to selectively culture neural stem/progenitor cells in the form of neurospheres, in which progenitor cells rather than stem cells are dominantly present. Moreover, inhibiting bone morphogenetic protein during the formation of EBs promotes differentiation into the neuroepithelium, and adding a low concentration of Noggin plays an important role in the formation of the forebrain. Adding a low concentration of retinoic acid, which is important in neural induction and the development of the hindbrain and anterior spinal cord, increases the proportion 
of neural stem/progenitor cells in the EBs and the efficiency of neurosphere formation.

Primary neurospheres (PNSs) formed in this way can be subcultured as secondary neurospheres (SNSs) and tertiary neurospheres. Intriguingly, although PNSs produce neurons almost exclusively, SNSs and tertiary neurospheres produce glial cells, including astrocytes and oligodendrocytes, as well as neurons. The ability of neurospheres to be subcultured repeatedly and to produce all 3 cell lineages of the nervous system demonstrates that mouse ES cellderived neurospheres contain neural stem/progenitor cells, as defined by their potential for self-renewal and their pluripotency. Moreover, the changes in differentiation potential associated with subculturing closely reflect the temporal changes seen in the development of the nervous system in vivo, in which only neurons are born in the initial stage of development, and glial cells first begin to be born in the middle stage of development. Furthermore, by adding Noggin or changing the concentration of retinoic acid during EB formation, neural stem/progenitor cells with anterior-posterior axis region-specific properties can be induced. Similarly, dorsal-ventral region-specific control has been achieved by adding the ventralization factor Sonic Hedgehog or the dorsalization factor bone morphogenetic protein 4 or Wnt3a to the culture during the formation of PNSs [22]. Based on these findings, adding the right factor at the right time during the culture appears to permit the region-specific control of neural stem/progenitor cell properties.

\section{Transplantation of Mouse ES Cell-Derived Neural Stem/Progenitor Cells into Injured Spinal Cords}

The self-renewal potential, pluripotency, and genetic adaptability of ES cells make them an attractive cell source for therapeutic transplantation. Methods of inducing mouse ES cells to form neurons have already been widely investigated [23], and ES cell-derived neural stem/progenitor cells seem ideal as a source of cells for transplantation. Furthermore, the differentiation stage of the transplanted cells can be specified, from undifferentiated ES cells to EBs to differentiated neurons. However, as the degree of differentiation decreases, the rate of teratoma formation by the transplanted cells increases [24].

In terms of the efficacy of ES cell transplantation for treating SCI, in 1999, McDonald et al. [25] formed EBs from mouse ES cells, and reported good functional recovery when they were transplanted into rats with a SCI. However, their study did not rule out the risk of tumor formation during a longer observation period or as a result of transplanting less-differentiated EBs. Keirstead et al. [26] established a method for efficiently inducing human ES cells to form very pure populations of oligodendrocyte progenitor cells by using a liquid culture medium that contained a factor (such as insulin or thyroid hormone) that promotes oligodendrocyte differentiation. They reported the remyelination of demyelinated axons and hind limb function recovery when they transplanted these cells into the injured spinal cords of rats. Later, Yamada et al. [27] formed EBs from ES cells and transplanted them into injured spinal cords, after applying an electrical stimulus to the EBs that caused the selective induction of neuronal differentiation. These authors found that a higher proportion of the electrically induced EBs differentiated into neurons than did control EBs. They also showed that the proliferative ability of the EBs that had been exposed to the electrical stimulus was lower than that of the EBs that were not exposed to an electrical stimulus. Therefore, they concluded that electrical stimulation was an effective method for safely inducing neuronal differentiation; however, it did not lead to the recovery of hind limb function after SCI. The stage at which neural progenitor cells induced from ES cells are most suitable for transplantation therapy in the subacute stage after injury was not clear from any of these studies.

In another report from our group, Kumagai et al. [28] used the culture system of Okada et al. [22], as previously described, to induce the formation of PNSs from mouse ES cells via EBs, and then of SNSs, by subculturing the PNSs. They transplanted PNSs and SNSs into a mouse spinal cord contusive injury model in the subacute stage, on day 9 after the injury, and conducted a study of their treatment efficacy. As previously stated, differentiation from the PNS cells was almost entirely into the neurons, whereas the SNS cells tended to differentiate into all 3 cell lineages (i.e., astrocytes and oligodendrocytes), as well as neurons. ${ }^{21}$ However, posttransplantation luciferase bioimaging of the PNSs and SNSs showed that approximately $20 \%$ of the cells had survived. Furthermore, the cells from the transplanted PNSs and SNSs tended to differentiate into all 3 nervous system cell lineages in vivo. The grafted PNSs and SNSs differentiated into $\mathrm{Hu}-$ positive neurons (PNS: $52.8 \pm 19.1 \%$; SNS: $16.3 \pm 5.2 \%$ ), glial fibrillary acidic protein (GFAP)-positive astrocytes (PNS: $19.0 \pm 9.3 \%$; SNS: $42.2 \pm 14.4 \%$ ), and Adenomatous Polyposis coli (APC)-positive oligodendrocytes (PNS: 14.8 \pm 7.1\%; SNS: $33.6 \pm 5.4 \%$ ). Values are means \pm SEM, respectively.

In addition, the atrophy and demyelination that followed the contusive injury were significantly mitigated in the SNS transplant group compared with the PNS transplant group, and more extensive angiogenesis within the injured spinal cord was observed in the SNS transplant group. Quantitative analysis of the transverse area of the spinal cord at the lesion epicenter was significantly larger in the SNS group $\left(3.2 \pm 1.2 \mathrm{~mm}^{2}\right)$ compared with the PNS group $(1.3 \pm$ 
$0.1 \mathrm{~mm}^{2}$ ). The evaluation of hind limb function on the Basso mouse scale showed that only the SNS transplant group had good functional recovery, which was statistically significant compared with the control group that received the vehicle only. The SNS transplant group exhibited 6.4 \pm 1.0 points on the Basso mouse scale, which represented the frequent or consistent plantar stepping with mostly coordinated paws parallel at initial contact, whereas the PNS control group showed $4.2 \pm 1.0$ points, which indicated the occasional plantar stepping [28].

Besides the reduction in atrophy and demyelination, and the increased angiogenesis, the functional recovery was apparently attributable to axon regrowth of the 5hydroxytryptamine-positive serotonergic raphespinal tract fibers, which are important for the motor functional recovery of hind limbs of rodents promoted by the transplanted cells. The functional recovery was also apparently attributable to remyelination by transplanted cells that differentiated into oligodendrocytes. Quantitative analysis of 5-HT-positive fibers in axial sections 4-mm caudal to the lesion epicenter resulted in significantly more 5-HT-positive fibers that were observed in the SNS grafted group $(0.0054$ $\left.\pm 0.002 \mathrm{~mm}^{2}\right)$ than the PNS grafted group $(0.0019 \pm$ $0.001 \mathrm{~mm}^{2}$ ) at 6 weeks postinjury. Significantly more Luxol fast blue-positive areas were detected in the SNS grafted group compared with the PNS grafted group at the lesion epicenter and 1-mm rostral and caudal to the lesion epicenter. These findings suggested that when applying ES cells to the treatment of SCI, it is preferable to transplant ES-derived cells that have formed neural stem/progenitor cells able to give rise to both glial cells and neurons, rather than neural stem/progenitor cells that generate almost only neurons. Thus, these findings are extremely important for future attempts to implement ES-cell and iPS-cell transplantation therapy.

However, even though aborted fetuses were not used to obtain these cells, which were established from excess embryos from infertility treatments, the ethical issues remain. The same is true of fetus-derived neural stem cells, but their use is even more problematic, because these cells would generally be used as allografts, which could trigger immunological rejection.

\section{iPS Cell-Derived Neural Stem Cells}

Assessment of the Safety of Using Mouse iPS Cell-Derived Neural Stem Cells

A breakthrough in regard to resolving the problems associated with using stem cells described thus far was provided by Professor Shinya Yamanaka and colleagues at Kyoto University, who established iPS cells from mouse and human fibroblasts in 2006 and 2007, respectively [15, 16]. iPS cells are somatic cells that have been reprogrammed by transducing such genes as Oct3/4, Sox2, Klf4, (and $c-M y c$ ) into mouse or human fibroblasts. They are pluripotent stem cells that have the same proliferative ability and differentiation potential as ES cells. It appears possible to generate iPS cells from the somatic cells of individual patients, a technology that is expected to resolve the ethical and immunological problems that hinder the use of ES cells. Nonetheless, some concerns regarding the use of iPS remain: 1) the potential introduction of transgenes (e. g., as a result of using viral vectors) and 2) a possible greater risk of tumor formation, even than with ES cells, if the fibroblasts are not completely reprogrammed.

We recently demonstrated great differences in the tumorigenicity and responsiveness to neural induction signals of iPS cells derived from various somatic tissues [29]. We generated 36 independent mouse iPS cell clones from various tissues and induced them to differentiate into neurospheres. Then we evaluated their differentiation capacity and post-transplantation safety in vivo by transplanting them into the corpus striatum of the brains of NOD/SCID mice. Almost all of the iPS cell clones could differentiate into neurospheres. However, detailed analyses of the cells by flow cytometry revealed that the percentages of undifferentiated Nanog-Enhanced Green Fluorescence Protein-positive cells present in the neurospheres after differentiation induction varied greatly with the source of the somatic cells that were reprogrammed into the iPS cells.

For example, iPS cell clones derived from mouse embryo fibroblasts (MEFs) showed equal responsiveness to neural differentiation-inducing signals as ES cells, and hardly any undifferentiated cells remained in the neurospheres. Post-transplantation teratoma formation in mice with transplants of MEF-iPS-cell-clone-derived neurospheres was both as infrequent and as minor as in the group transplanted with ES cell-derived neurospheres. Moreover, no teratoma formation was observed during a 16-week observation period of mice with transplants of 2 iPS cell clones derived from adult gastric epithelial cells (Stm) [30].

By contrast, iPS cell clones derived from adult tail-tip fibroblasts (TTFs) displayed statistically significant resistance to differentiation, and many undifferentiated cells remained in the neurospheres after the induction of differentiation. Furthermore, the TTF-iPS-derived neurospheres formed significantly larger teratomas when transplanted into NOD/SCID mice, many of which soon became debilitated or died. Neurospheres derived from iPS cell clones from the adult liver, (Hep)-derived iPS cells, were intermediate between the MEF-iPS cell clones and TTF-iPS cell clones for both responsiveness to neural differentiation signals and tumorigenesis. 
Interestingly, neither the transduction of $c-M y c$ nor screening of the reprogrammed cells with a reporter molecule was associated with the responsiveness of the iPS cells to the induction of differentiation or with their safety after transplantation. It appears that the previously described persistence of gene-expression patterns of the original somatic cells from which the iPS cells were derived may explain the variations in their differentiation capacity, but an urgent need remains for a detailed analysis of the properties of iPS cells derived from different tissues.

\section{Transplantation of "Safe" Mouse iPS Cell-Clone-Derived Neural Stem Cells to Treat SCI}

In light of the previously described studies in relation to safety, we first assessed the safety of each clone by transplanting neurospheres into the brain of the NOD/SCID mice, also as previously described. Next, we used a mouse iPS cell clone whose safety had been confirmed (38 C2 clone), to study the effectiveness of treating SCI by transplanting neurospheres derived from it into the lesion site in a mouse model [31]. The 38 C2-iPS-derived secondary neurosphere (38 C2-SNS) transplantation was performed in the subacute stage on day 9 after the injury. In a similar way, as with the previously mentioned experiments with derived ES cells, the results of luciferase bioimaging showed that approximately $20 \%$ of the transplanted 38 C2-SNSs survived within the injured spinal cord, and also showed that they differentiated into neurons $(31.4 \pm 1.1 \%)$, astrocytes $(49.3 \pm 4.5 \%)$, and oligodendrocytes $(14.4 \pm 3.0 \%)$, suggesting that almost all of the grafted 38 C2-SNSs differentiated into definable CNS cell types. Severe atrophic change and demyelination had occurred in the injured spinal cord after the contusive injury, but the changes were significantly mitigated in the group treated with the 38 C2-SNSs. Quantification of Luxol fast bluepositive areas at the lesion epicenter 42 days after injury revealed that the $38 \mathrm{C} 2$-SNS grafted mice showed a significantly larger myelinated area than the PBS control mice (38 C2-NS, $0.19 \pm 0.029 \mathrm{~mm}^{2}$; controls, 0.046士 $0.012 \mathrm{~mm}^{2}$; values are mean $\pm \mathrm{SEM} ; n=7$ each). Moreover, in the $38 \mathrm{C} 2$-SNS grafted group, the serotonergic innervation of the distal cord compared to the control animals was promoted and supported by grafted 38 C2-SNS-derived glial fibrillary acidic protein ${ }^{+}$astrocytes, which exhibited a bipolar morphology with long processes extending along the axis of the spinal cord, caudal to the lesion epicenter in close association with $5-\mathrm{HT}^{+}$host serotonergic fibers, thereby enhancing functional recovery after SCI [31]. Quantitative analysis of the serotonergic innervation of the distal cord revealed a significant difference between the 38 C2-SNS $\left(0.0051 \pm 0.0010 \mathrm{~mm}^{2}\right)$ and the PBS control groups $\left(0.0019 \pm 0.00021 \mathrm{~mm}^{2}\right)$. The contusive injury (60 kdyn) resulted in a significant decrease in the number of 5$\mathrm{HT}^{+}$fibers at the distal cord followed by a slight recovery, which is a nature of contusive SCI. The injection of PBS in the PBS control group did not induce any additional increase in the number of $5-\mathrm{HT}^{+}$fibers at the distal cord. In contrast, innervation of the distal cord by these $5-\mathrm{HT}^{+}$ fibers was enhanced by the grafted 38 C2-SNS 6 weeks after SCI [31]. Evaluation of the motor function of the hind limbs on the Basso mouse scale showed significantly better recovery of function in the group treated with iPS-cellderived neurospheres than in the control groups (PBStreated group and fibroblast-transplanted group). Forty-two days after injury, the $38 \mathrm{C} 2$-SNS-grafted animals could lift their trunks (BMS points, $4.3 \pm 0.28$ ) and had significantly better BMS than the PBS control animals (BMS points, 2.8 \pm 0.2 ), which were unable to support their body weight with their hind limbs. From a clinical perspective, the recovery of weight-supported plantar steps in the $38 \mathrm{C} 2$-SNS grafted group was noteworthy. This functional recovery was apparently attributable to effects, such as the promotion of axonal regrowth in the raphespinal tract supported by graftderived bipolar astrocytes and the remyelination by the transplanted cells (Fig. 1), in addition to the mitigation of the atrophic change and demyelination previously mentioned. However, this promotion of serotonergic innervation at the distal cord was not the direct effect of grafted $38 \mathrm{C} 2-$ SNSs (i.e., the noncell autonomous effect of grafts).

\section{Adult Tissue-Derived "Safe" iPS Cell Clones and "Unsafe" iPS Cell Clones}

Next, we conducted a similar transplantation experiment using adult-tissue (TTF)-derived iPS cells as a model that was closer to actual clinical application. There were 6 TTFderived clones among the 36 mouse iPS cell clones used in our joint safety study with Miura et al. [29], but only one of them, clone 335D1, was confirmed to be safe in our teratoma assay. We used clone 335D1 along with 2 "unsafe" clones $(256 \mathrm{H} 13$ and $256 \mathrm{H} 18)$ in a transplantation experiment, in which we induced the clones to form neurospheres and transplanted them into the mouse SCI model, as described for clone $38 \mathrm{C} 2$.

We found the improvement to be suddenly lost, although functional recovery was achieved after transplantation of the neurospheres from all of the clones $(335 \mathrm{D} 1,256 \mathrm{H} 13$, 256H18) into the injured spinal cord 6 weeks after the injury in the mice, with transplants of the "unsafe" clone-derived neurospheres. Most of these mice then suddenly died.

A histological analysis revealed huge teratomas within the spinal cords of the animals that had been treated with neurospheres derived from the "unsafe" 
Fig. 1 Immunohistochemistry of 38 C2-mouse embryo fibroblast-induced pluripotent stem-subcultured as secondary neurospheres-derived mature oligodendrocytes (Myelin Basic Protein+). Grafted cells (Red Fluorescence Protein+) were integrated into myelin sheath and differentiated into myelinating oligodendrocytes. Scale bar $=20 \mu \mathrm{m}$
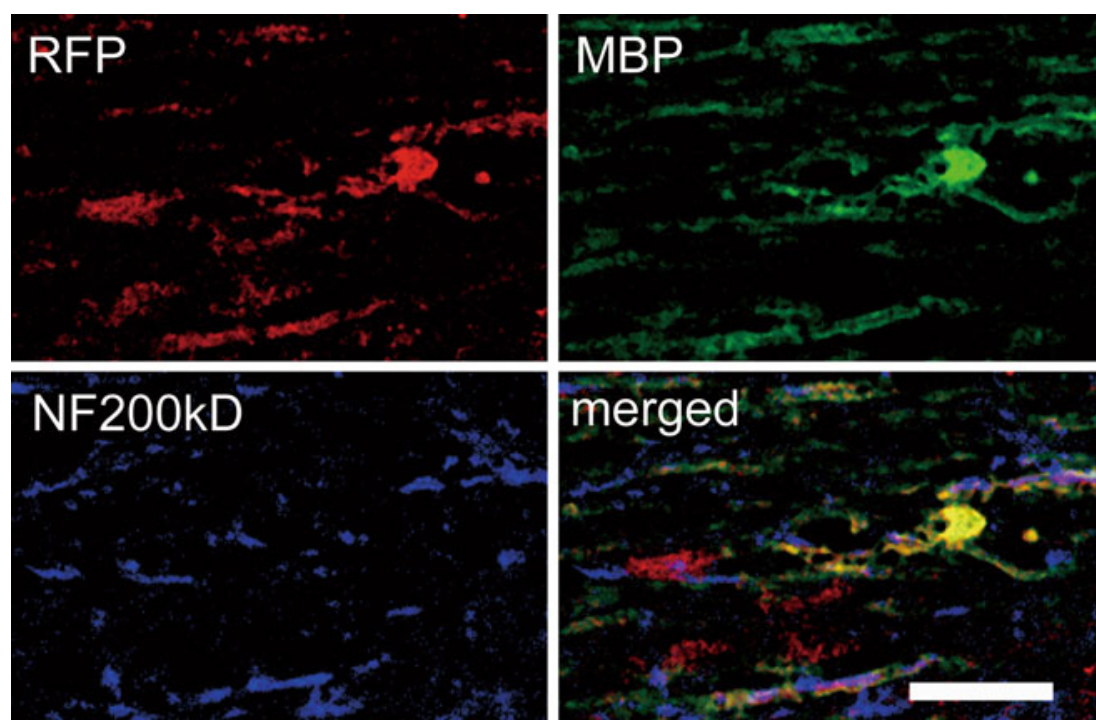

clones. Moreover, these grafted "unsafe" cells exhibited Nanog and Oct3/4 immunoreactivity (Fig. 2), suggesting that the grafted and surviving "unsafe" clone-derived cells were in the undifferentiated state, and failed to differentiate the definable CNS cell types, including neurons, astrocytes and oligodendrocytes, and resulted in forming teratoma within the injured cord. By contrast, when the "safe" 335D1 clone was used, no tumor formation was observed in any of the mice, as expected, and their functional recovery was statistically significant in comparison with the control group and equivalent to that obtained with ES cell-derived neurospheres.

These findings showed that adult tissue-derived iPS cell clones were not as safe as clones derived from embryonic tissue, and although there was extremely wide variation among the clones in relation to their safety after transplantation, if their safety is rigorously assessed in advance, they

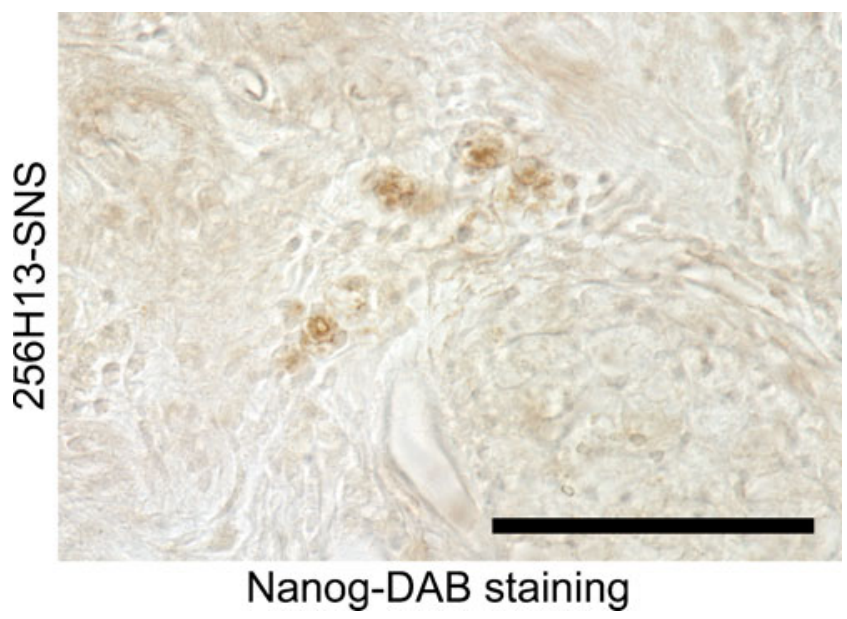

Fig. 2 Scattered small clusters of Nanog+undifferentiated cells were observed in these mice without obvious teratoma formation. Scale bar= $100 \mu \mathrm{m}$. diaminobenzine $=\mathrm{xxxx}$ may be a useful source of cells for the treatment of SCI [31].

\section{Future Prospects and Tasks}

As previously indicated, iPS cells may have great potential as a source of cells for autologous transplantation. In recent years, it has become possible to generate human iPS cells from a single drop of blood [32], and iPS cell research is advancing rapidly. However, retroviruses and lentiviruses are used to transduce reprogramming factors during iPS cell establishment, and because these viruses are often integrated in the vicinity of gene promoters, there is a risk of altering the pattern of expression of nearby endogenous genes and causing tumor formation. Actual examples of leukemia developing in 2 of 10 patients with X-linked severe combined immunodeficiency who underwent gene therapy with a retrovirus vector have been reported [33]. Fortunately, in recent years, iPS cells have been successfully produced without using retroviruses or lentiviruses by means of transient gene expression [32, 34-36] and protein transduction [37, 38], and by replacing some of the previously transduced genes with drug treatment [39, 40]. The use of iPS cells produced without inserting exogenous genes appears to be preferable for cell transplantation therapy. However, detailed comparisons of the properties of iPS clones generated by these various methods will be necessary to determine whether their pluripotency and in vitro differentiation capacity are comparable to those of iPS cells produced with retroviruses. Furthermore, the issue of genomic instability, including copy number variations and whole chromosome aneuploidies are very important in considering the safety issue of iPS cells-based therapy, irrespective to the methods of iPS cells establishment. 
Moreover, when we turn our attention to the pathology of the SCI itself, we find that many tasks must still precede the use of iPS cells in clinical applications. Three main mechanisms by which the transplanted cells contribute to the recovery of function have been proposed as follows: 1) synaptic formation and axon regeneration by the transplanted cells, 2) remyelination by the transplanted cells, and 3) trophic support by the transplanted cells [41]. Detailed investigations and verification of these 3 mechanisms seem essential to achieving the goal of clinical application, but it must be said that, even in actual clinical practice, many aspects of the pathology of SCI itself, including the fact that the paralysis in the hyperacute stage and prediction of the outcome are not always the same, and remain obscure. Not being able to evaluate minute changes in the injured spinal cord tissue of living animals in the span of time appears to be one of the factors that stands in the way of a breakthrough, but we have been pursuing research that should allow us to accomplish this task 1 step at a time. We have recently focused on anisotropic diffusion in the spinal cord white matter, and succeeded in establishing diffusion tensor tractography, which is a novel axon-specific evaluation system that uses magnetic resonance imaging [42], and we are attempting to apply this technology to patients with spinal injuries and spinal cord disease (Fig. 3).

Research on human ES cells, under strict supervision, is also currently being pursued in Japan. Studies on culture methods for human ES cells and iPS cells have already begun in our laboratory; however, not surprisingly, we face the same problems when using human ES cells as needed to be resolved when using mouse ES cells. The most
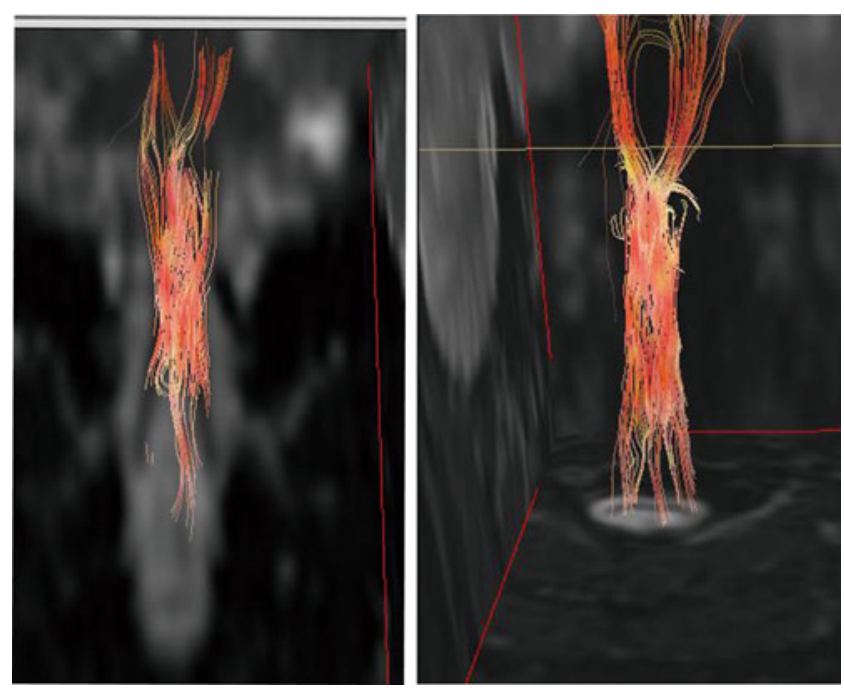

Fig. 3 Diffusion tensor tractography images of the injured cervical spinal cord of a patient, made using 1.5 Tesla magnetic resonance imaging. The man injured his spinal cord during a skiing accident 27 years before. White matter tracts were disrupted at the injury site, and the disruption is visible on the image important problem is safety, especially for clinical applications, because the intermingling of undifferentiated ES/iPS cells with differentiated cells might cause teratoma formation, and new methods are needed to only obtain the desirable cells. Two approaches that have been considered are to select the appropriate cells with $100 \%$ reliability, by inserting a drug-resistance gene or a marker gene under a lineage-specific promoter [43], and to kill only highly proliferative cells by introducing the drug-inducible expression of a gene that governs cell death [44]. However, this is a difficult problem, and although the task is urgent, it will be some time before it is possible to guarantee sufficient safety to enable stem cell use in clinical settings. Prior to any clinical trial of human CNS disorders using iPS cells, it will be essential to pre-evaluate each iPS cell clone carefully to be able to guarantee a safety level equal to other types of cells, such as hair follicle stem cells [45-51], multipotent skin-derived precursor cells [52, 53], fetusderived neural stem/progenitor cells [54], and neural crest stem cells [55]. Moreover, experiments on primates will be essential, as preclinical studies before transplantation can be tried in SCI patients [56]. In the future, after rigorously evaluating its safety, we plan to conduct further studies in our own laboratory using human ES/iPS-cell-derived neural stem/progenitor cell transplantation in a primate SCI model.

At the beginning of 2009, the United States Food and Drug Administration (FDA) approved a clinical trial (phase 1) in the United States of Geron Corporation's ES cellderived oligodendrocyte progenitor cells to treat acute stage SCI, which has attracted a great deal of worldwide attention. However, the use of human ES cells is problematic among people with certain ethical and religious viewpoints. iPS cells have attracted a great deal of attention as a technology that can resolve these problems, but the difficulties that currently prevent the use of these cells in the clinic, and the needed investigations, including developing new methods of inducing the cells, establishing their tumorigenicity, and so forth, must first be resolved with human ES cells. ES cell research can be described as the "other leg" of iPS cell research, and even from the standpoint of aiming at future clinical applications, it seems absolutely essential to pursue research on both of them simultaneously. Although a recent article indicates a stronger immunogenicity of the mouse iPS cells than that of mouse ES cells at transplantation of these undifferentiated mouse pluripotent stem cells into the $\mathrm{C} 57 \mathrm{BL} / 6$ mice [57], our previous results indicate that mouse iPS cellderived neural stem/progenitor cells [31] survived in a similar way as that of mouse ES cell-derived neural/stem progenitor cells [28] and mouse fetal CNS-derived neural/ stem progenitor cells [13] when transplanted into the SCI model of $\mathrm{C} 57 \mathrm{BL} / 6$ mice. Thus, the issue of the immunogenicity of human iPS cell-derived cells should be 
investigated in comparison with human ES cell-derived cells and human somatic stem cells before the clinical application.

Acknowledgments The original investigation on iPS cells was performed in collaboration with Professor Shinya Yamanaka (Center for iPS Cell Research \& Application, Kyoto University, Japan). This work was supported by grants from Grants-in-Aid for Scientific Research from JSPS and the Ministry of Education, Culture, Sports, Science and Technology of Japan (MEXT), the project for realization of regenerative medicine and Support for the core institutes for iPS cell research from MEXT; Research Fellowships for Young Scientists from the Japan Society for the Promotion of Science; Keio Gijuku Academic Development Funds; and by a Grant-in-aid for the Global COE program from MEXT to Keio University.

Full conflict of interest disclosure is available in the electronic supplementary material for this article.

\section{References}

1. Ramon y Cajal S. 1928. Degeneration and Regeneration of the Nervous System. New York: Hafner.

2. Reynolds BA, Weiss S. Generation of neurons and astrocytes from isolated cells of the adult mammalian central nervous system. Science 1992;255:1707-1710.

3. Kilpatrick TJ, Bartlett PF. Cloning and growth of multipotential neural precursors: requirements for proliferation and differentiation. Neuron 1993;10:255-265.

4. Davis AA, Temple S. A self-renewing multipotential stem cell in embryonic rat cerebral cortex. Nature 1994;372:263-266.

5. Palmer TD, Ray J, Gage FH. FGF-2-responsive neuronal progenitors reside in proliferative and quiescent regions of the adult rodent brain. Mol Cell Neurosci 1995;6:474-486.

6. Doetsch F, Caille I, Lim DA, Garcia-Verdugo JM, Alvarez-Buylla A. Subventricular zone astrocytes are neural stem cells in the adult mammalian brain. Cell 1999;97:703-716.

7. Alvarez-Buylla A, Garcia-Verdugo JM, Tramontin AD. A unified hypothesis on the lineage of neural stem cells. Nat Rev Neurosci 2001;2:287-293.

8. Seri B, Garcia-Verdugo JM, McEwen BS, Alvarez-Buylla A. Astrocytes give rise to new neurons in the adult mammalian hippocampus. J Neurosci 2001;21:7153-160.

9. van Praag H, Schinder AF, Christie BR, Toni N, Palmer TD, Gage FH. Functional neurogenesis in the adult hippocampus. Nature 2002;415:1030-1034.

10. Eriksson PS, Perfilieva E, Bjork-Eriksson T, et al. Neurogenesis in the adult human hippocampus. Nat Med 1998;4:1313-1317.

11. Bregman BS, Kunkel-Bagden E, Reier PJ, Dai HN, McAtee M, Gao D. Recovery of function after spinal cord injury: mechanisms underlying transplant-mediated recovery of function differ after spinal cord injury in newborn and adult rats. Exp Neurol 1993;123:3-16.

12. Ogawa Y, Sawamoto K, Miyata T, et al. Transplantation of in vitroexpanded fetal neural progenitor cells results in neurogenesis and functional recovery after spinal cord contusion injury in adult rats. J Neurosci Res 2002;69:925-933.

13. Okada S, Ishii K, Yamane J, et al. In vivo imaging of engrafted neural stem cells: its application in evaluating the optimal timing of transplantation for spinal cord injury. FASEB J 2005;19:18391841.

14. Iwanami A, Kaneko S, Nakamura M, et al. Transplantation of human neural stem cells for spinal cord injury in primates. J Neurosci Res 2005;80:182-190.
15. Takahashi K, Yamanaka S. Induction of pluripotent stem cells from mouse embryonic and adult fibroblast cultures by defined factors. Cell 2006;126:663-676.

16. Takahashi K, Tanabe K, Ohnuki M, et al. Induction of pluripotent stem cells from adult human fibroblasts by defined factors. Cell 2007;131:861-872.

17. Yu J, Vodyanik MA, Smuga-Otto K, et al. Induced pluripotent stem cell lines derived from human somatic cells. Science 2007;318:1917-1920.

18. Hitoshi S, Seaberg RM, Koscik C, et al. Primitive neural stem cells from the mammalian epiblast differentiate to definitive neural stem cells under the control of Notch signaling. Genes Dev 2004;18:1806-1811.

19. Miyata T, Kawaguchi A, Okano H, Ogawa M. Asymmetric inheritance of radial glial fibers by cortical neurons. Neuron 2001;31:727-741.

20. Noctor SC, Flint AC, Weissman TA, Dammerman RS, Kriegstein AR. Neurons derived from radial glial cells establish radial units in neocortex. Nature 2001;409:714-720.

21. Temple S. The development of neural stem cells. Nature 2001;414:112-117.

22. Okada Y, Matsumoto A, Shimazaki T, et al. Spatiotemporal recapitulation of central nervous system development by murine embryonic stem cell-derived neural stem/progenitor cells. Stem Cells 2008;26:3086-3098.

23. Bibel M, Richter J, Schrenk K, et al. Differentiation of mouse embryonic stem cells into a defined neuronal lineage. Nat Neurosci 2004;7:1003-1009.

24. Brederlau A, Correia AS, Anisimov SV, et al. Transplantation of human embryonic stem cell-derived cells to a rat model of Parkinson's disease: effect of in vitro differentiation on graft survival and teratoma formation. Stem Cells 2006;24:1433-1440.

25. McDonald JW, Liu XZ, Qu Y, et al. Transplanted embryonic stem cells survive, differentiate and promote recovery in injured rat spinal cord. Nat Med 1999;5:1410-1412.

26. Keirstead HS, Nistor G, Bernal G, et al. Human embryonic stem cell-derived oligodendrocyte progenitor cell transplants remyelinate and restore locomotion after spinal cord injury. J Neurosci 2005;25:4694-4705.

27. Yamada M, Tanemura K, Okada S, et al. Electrical stimulation modulates fate determination of differentiating embryonic stem cells. Stem Cells 2007;25:562-570.

28. Kumagai G, Okada Y, Yamane J, et al. Roles of ES cell-derived gliogenic neural stem/progenitor cells in functional recovery after spinal cord injury. PLoS One 2009;4:e7706.

29. Miura K, Okada Y, Aoi T, et al. Variation in the safety of induced pluripotent stem cell lines. Nat Biotechnol 2009;27:743-745.

30. Aoi T, Yae K, Nakagawa M, et al. Generation of pluripotent stem cells from adult mouse liver and stomach cells. Science 2008;321:699-702.

31. Tsuji O, Miura K, Okada Y, et al. Therapeutic potential of appropriately evaluated safe-induced pluripotent stem cells for spinal cord injury. Proc Natl Acad Sci U S A. 2010 Jul 13;107 (28):12704-12709. Epub 2010 Jul 6.

32. Seki T, Yuasa S, Oda M, et al. Generation of induced pluripotent stem cells from human terminally differentiated circulating $\mathrm{T}$ Cells. Cell Stem Cell 2010;7:11-14.

33. Hacein-Bey-Abina S, Von Kalle C, Schmidt M, et al. LMO2associated clonal $\mathrm{T}$ cell proliferation in two patients after gene therapy for SCID-X1. Science 2003;302:415-419.

34. Yu J, Hu K, Smuga-Otto K, et al. Human induced pluripotent stem cells free of vector and transgene sequences. Science 2009;324:797-801.

35. Kaji K, Norrby K, Paca A, Mileikovsky M, Mohseni P, Woltjen K. Virus-free induction of pluripotency and subsequent excision of reprogramming factors. Nature 2009;458:771-775. 
36. Woltjen K, Michael IP, Mohseni P, et al. piggyBac transposition reprograms fibroblasts to induced pluripotent stem cells. Nature 2009;458:766-770.

37. Zhou H, Wu S, Joo JY, et al. Generation of induced pluripotent stem cells using recombinant proteins. Cell Stem Cell 2009; 4:381-384.

38. Kim D, Kim CH, Moon JI, et al. Generation of human induced pluripotent stem cells by direct delivery of reprogramming proteins. Cell Stem Cell 2009;4:472-476.

39. Shi Y, Do JT, Desponts C, Hahm HS, Scholer HR, Ding S. A combined chemical and genetic approach for the generation of induced pluripotent stem cells. Cell Stem Cell 2008;2:525-528.

40. Maherali N, Hochedlinger K. Tgfbeta signal inhibition cooperates in the induction of iPSCs and replaces Sox 2 and cMyc. Curr Biol 2009;19:1718-1723.

41. Barnabe-Heider F, Frisen J. Stem cells for spinal cord repair. Cell Stem Cell 2008;3:16-24.

42. Fujiyoshi K, Yamada M, Nakamura M, et al. In vivo tracing of neural tracts in the intact and injured spinal cord of marmosets by diffusion tensor tractography. J Neurosci 2007;27:1199111998.

43. Marchetti S, Gimond C, Iljin K, et al. Endothelial cells genetically selected from differentiating mouse embryonic stem cells incorporate at sites of neovascularization in vivo. J Cell Sci 2002;115:2075-2085.

44. Schuldiner M, Itskovitz-Eldor J, Benvenisty N. Selective ablation of human embryonic stem cells expressing a "suicide" gene. Stem Cells 2003;21:257-265.

45. Li L, Mignone J, Yang M, et al. Nestin expression in hair follicle sheath progenitor cells. Proc Natl Acad Sci U S A 2003;100: 9958-9961.

46. Amoh Y, Hamada Y, Aki R, Kawahara K, Hoffman RM, Katsuoka K. Direct transplantation of uncultured hair-follicle pluripotent stem (hfPS) cells promotes the recovery of peripheral nerve injury. J Cell Biochem 2010;110:272-277.
47. Amoh Y, Kanoh M, Niiyama S, et al. Human hair follicle pluripotent stem (hfPS) cells promote regeneration of peripheralnerve injury: an advantageous alternative to ES and iPS cells. $J$ Cell Biochem 2009;107:1016-1020.

48. Amoh Y, Li L, Campillo R, et al. Implanted hair follicle stem cells form Schwann cells that support repair of severed peripheral nerves. Proc Natl Acad Sci U S A 2005;102:17734-17738.

49. Amoh Y, Li L, Katsuoka K, Hoffman RM. Multipotent hair follicle stem cells promote repair of spinal cord injury and recovery of walking function. Cell Cycle 2008;7:1865-1869.

50. Amoh Y, Li L, Katsuoka K, Penman S, Hoffman RM. Multipotent nestin-positive, keratin-negative hair-follicle bulge stem cells can form neurons. Proc Natl Acad Sci U S A 2005;102:5530-5534.

51. Liu F, Uchugonova A, Kimura $\mathrm{H}$, et al. The bulge area is the major hair follicle source of nestin-expressing pluripotent stem cells which can repair the spinal cord compared to the dermal papilla. Cell Cycle 2011;10:830-839.

52. Biernaskie J, Sparling JS, Liu J, et al. Skin-derived precursors generate myelinating Schwann cells that promote remyelination and functional recovery after contusion spinal cord injury. J Neurosci 2007;27:9545-9559.

53. Fernandes KJ, McKenzie IA, Mill P, et al. A dermal niche for multipotent adult skin-derived precursor cells. Nat Cell Biol 2004;6:1082-1093.

54. Lindvall O, Kokaia Z, Martinez-Serrano A. Stem cell therapy for human neurodegenerative disorders-how to make it work. Nat Med 2004;10(suppl):S42-S50.

55. Nagoshi N, Shibata S, Kubota Y, et al. Ontogeny and multipotency of neural crest-derived stem cells in mouse bone marrow, dorsal root ganglia, and whisker pad. Cell Stem Cell 2008;2:392-403.

56. Iwanami A, Yamane J, Katoh H, et al. Establishment of graded spinal cord injury model in a nonhuman primate: the common marmoset. J Neurosci Res 2005;80:172-181.

57. Zhao T, Zhang ZN, Rong Z, Xu Y. Immunogenicity of induced pluripotent stem cells. Nature 2011 May 13;474(7350):212-215. 